\title{
STELLAR EVOLUTION AND DYNAMICS IN STAR CLUSTERS
}

\author{
SIMON F. PORTEGIES ZWART \\ Astronomical Institute Anton Pannekoek, Kruislaan 403, NL-1098 SJ Amsterdam \\ CHRISTOPHER A. TOUT \\ Department of Mathematics, Monash University, Clayton, Victoria 3168, Australia. \\ AND \\ HYUNG MOK LEE \\ Dept. of Earth Sciences, Pusan National University, Pusan 609-735, Korea
}

\begin{abstract}
Dynamical models of star clusters are maturing in the sense that effects other than simple point particle dynamics are taken into account. We summarize the relevance of, and prospects for, this new generation of $N$-body models.
\end{abstract}

\section{Summary and Introduction}

Realistic star clusters are not born instantly nor are they isolated from external perturbations nor do they consist of single time-independent equal-mass points. On the contrary a realistic star cluster is born gradually from a contracting gas cloud, which is embedded in the external potential of a galaxy, and consists of evolving single stars as well as multiple systems. Owing to lack of computer power and partially to lack of software, computations, in which all these effects are accounted for, have not yet been performed.

However numerical models of star clusters are beginning to come of age and incorporate deviations from the ideal star cluster. Some models already include mutual interaction between stellar evolution and star cluster dynamics (see Chernoff \& Weinberg 1990; Fukushige \& Heggie 1995; de la Fuente Marcos 1996; Spurzem \& Aarseth 1996; Einsel \& Spurzem 1997; Portegies Zwart et al. 1997; Tout et al. 1997, and the reviews Hut et al. 1992 and Meylan \& Heggie 1997). Here we study the arguments for combining stellar evolution and stellar dynamics in hybrid models, the advantages and disadvantages of performing such model computations and outline the future of dynamical models of star clusters.

\section{From zero to first order modelling}

Perturbations of the evolution of dynamical star clusters can be subdivided into two classes: those which affect the cluster's evolution from outside and are not significantly affected by the evolution of the star cluster, and those which affect it from inside and actually live in symbiosis with it undergoing mutual interaction. This mutual interaction can be thought of as the ecology of the star cluster (see Heggie 1992).

\subsection{THE TIMESCALE ARGUMENT}

The internal evolution of a stellar system is governed by two fundamental time scales: these are the crossing time $t_{\mathrm{crss}}=r_{\mathrm{vir}} /\langle v\rangle$, the ratio of the cluster virial radius to the mean velocity of its components, and the relaxation time $t_{\mathrm{rlx}}$. The ratio of these $t_{\mathrm{rlx}} / t_{\mathrm{crss}} \equiv n_{\mathrm{rlx}}$ is roughly proportional to $N$. In real globular clusters $N \approx 10^{5}$ and both timescales are usually well separated by more than three orders of magnitude.

Stimulated evolution of the star cluster such as stellar evolution or the external potential of the Galaxy introduces new time scales. It is generally the shortest timescale which drives the stimulated 


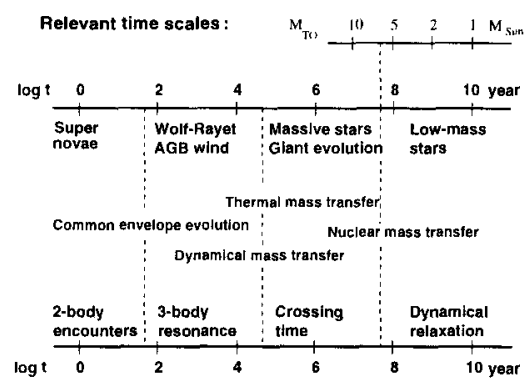

Figure 1. Time bar for the turn-off mass $M_{\mathrm{TO}}$ of star clusters (upper bar), for the evolution of single stars (second bar) and for the dynamical time scales of star clusters (lower bar). Those for the evolution of close binaries are indicated in between.

cvolution of the cluster and its stars so we must model these complex systems with a sufficiently fine time resolution. Figure 1 gives an overview of the dominant timescales in a star cluster

The evolution timescale for low-mass stars $\left(M \lesssim 1 \mathrm{M}_{\odot}\right)$ exceeds the age of globular clusters so that the long term evolution can be investigated with simple, few-component models containing main-sequence stars, white dwarfs and neutron stars (Lee 1987, Kim et al. 1998). The neutron stars, which are retained in these models, strongly affect the dynamics of the stellar system; the mass of white dwarfs is similar to that of normal stars while massive black holes are easily ejected by the interaction with other cluster black holes and binaries (Kulkarni et al. 1993). These first-order models already demonstrate the importance of the interaction between the dynamics of the star clusters and its static stellar population. They are useful for studying the future evolution of star clusters but their past requires more refined models.

\section{Stimulated evolution}

Higher-order models incorporate the details of stimulated evolution directly. Processes which stimulate the evolution of the star cluster fall into two categories, those which are affected by the internal evolution of the host cluster and those which are not.

\subsection{EXTERNAL STIMULATION}

Star clusters are relatively low-mass entities in an orbit around the much more massive Galaxy. A globular cluster is embedded in its effective Roche-lobe which limits the size of the cluster to its tidal radius. The timescale for a galactic revolution is of the order of $10^{8}$ to $10^{9} \mathrm{yr}$. Even if the galactocentric orbit is circular the effect of the tidal field is, owing to the characteristic pear shape of the Roche-lobe, not spherically symmetric. The Coriolis force on the circulating cluster and its fall through the galactic disc, twice per orbit, are expected to affect the dynamical evolution of the star cluster considerably. These effects are relatively straightforward to incorporate in dynamical models because there is no mutual interaction (i.e. second order effects): the cluster does not affect the evolution of the galactic disc. Molecular clouds and neighbouring star clusters are possibly affected by a passing cluster but this stimulated evolution is not coupled back to the host cluster (Spurzem \& Giersz 1996).

\subsection{INTERNAL STIMULATION}

A star cluster which consists of static equal-mass points is only affected by its internal dynamical evolution and external stimulated evolution. Once we relax the equal-mass assumption and use a more realistic mass function we must also include stellar evolution. In the past the mass function has been replaced by the equal-mass approximation partly because of this requirement but also because high-mass stars, which are considered to be the most interesting, are very rare compared to the low-mass objects which absorb most of the computer time. 


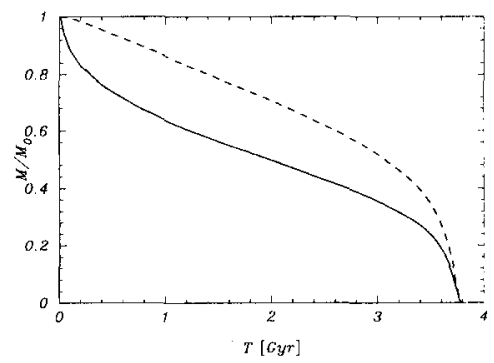

Figure 2. The time evolution of the total mass (solid line) and number of stars (dashed line) of the cluster for a standard, 32768-body run. Both the mass and the number of stars are normalized to their initial value (model M32 of Portegies Zwart et al. 1998, in prep). The initial star cluster contains 32768 stars chosen from a power-law initial mass function with exponent 2.5 (Salpeter $=2.35$ ) between $0.4 \mathrm{M}_{\odot}$ and $14 \mathrm{M}_{\odot}$. The cluster is situated in a circular orbit at a distance of about $15 \mathrm{kpc}$ from the Galactic centre.

The most massive stars $\left(m_{\star} \geq 10 M_{\odot}\right)$ evolve on a timescale comparable to the crossing time of a globular cluster. If such stars maintain the same mass and radius throughout the dynamical evolution of the stellar system, they sink to the cluster core by dynamical friction (free-free scattering) on a timescale $t_{\mathrm{df}} \propto t_{\mathrm{rl} x}\langle m\rangle / m_{\star}$ (of the order of $10^{8} \mathrm{yr}$ ) and begin to dominate the dynamics there. In reality such stars lose most of their mass within a few crossing times.

Figure 2 gives as a function of time the total mass and the total number of stars (normalized to its initial value) for a simulated star cluster. The figure demonstrates the dominant effect of mass loss by stellar evolution in the first few $10^{8} \mathrm{yr}$ followed by a more gradual decrease of mass as the most massive stars burn out and the mass loss is driven by stellar escapees. The final epoch is dominated by escaping stars as the stellar system cvaporates.

\section{Primordial binaries}

Primordial binaries are crucial for the dynamical evolution of a dense star cluster; their binding energies are an important reservoir that effectively heats the stellar system, preventing its core from collapsing. Including them in the computations is crucial, but ignoring the evolution of the binary components and together with that, the evolution of the binary parameters, is unrealistic. The binaries are most strongly coupled to the evolution of the star cluster (Hut 1994) and the variation in the binary parameters, owing to internal dynamical evolution, can have a dramatic effect on the dynamical evolution of the cluster as a whole.

The orbital parameters of binaries in clusters are affected by internal evolution and by subsequent dynamical encounters; this may lead to formation of objects which are difficult to explain by evolution of isolated binaries. The study of such stars in globular clusters can provide observational signatures of the relative importance of different dynamical and evolutionary processes. We describe some of these signatures in the following section.

\section{Outlook for full models}

At present there are two $N$-body models which incorporate such effects, Aarseth's NB0DY5 (Tout et al. 1997) and the hybridization of Kira (Hut et al 1995) and SeBa (Portegies Zwart \& Verbunt $1996)$ in starlab. Both these models have reached the end of their initial testing and are ready for computation of realistic but small chusters $\left(<10^{5}\right.$ stars). A direct comparison of the two using the same initial conditions will be one of the first major projects to be tackled. Because these are full models of both the position and velocity and the evolutionary state of ach individual stellar component it is possible to simulate observations for comparison with real clusters. Figure 3 illustrates how we can synthesize a ced image of a distant cluster as seen with an appropriate: telescope. With velocity information it is no more difficult to generate a Doppler map. In addition all the information exists to follow the integrated luminosity and colours and by including atmospheres for each star integrated cluster spectra can be produced. Because we follow the evolution of the 


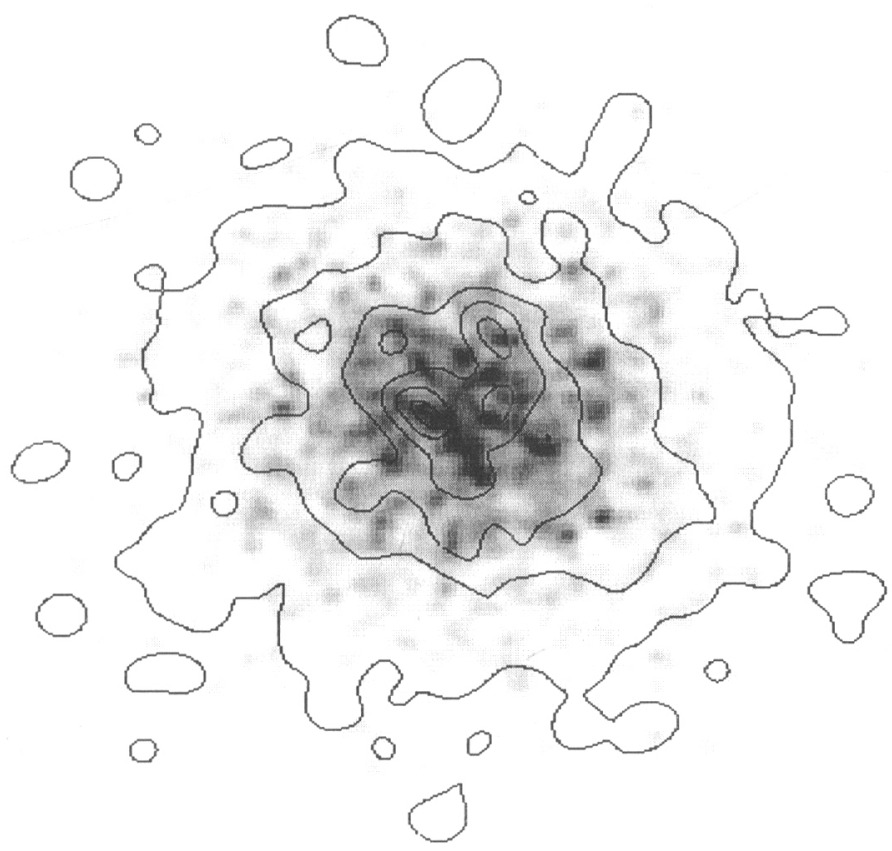

Figure 3. Picture of a synthesized globular stars cluster in visual magnitude overlayed with a synthesized radio mup. Because the luminosity, temperature, angular size, mass etc. of all stars in the simulation model are known it is straight forward to produce a picture of the system as if observed by a telescope with finite seeing. The picture is taken from the star cluster in fig. 2 at an age of $\sim 1 \mathrm{Gyr}$, at which point it still contains about 28000 stars of which about 250 are neutron stars.

whole cluster over its entire lifetime we can also calculate the evolution of any of these observable quantities for any chosen spatial region. Our only limitation at present is the total number of stars we can include. We are not yet able to compute the dynamics of millions of stars in a realistic globular cluster within a reasonable time. Future generations of special purpose computers (see Makino, these proceedings) will eventually make this possible and we note here that there will be no problem including detailed stellar evolution in such models because the time required increases only linearly with the number of stars and interacting binaries.

These combined models allow the investigation of the detailed evolution of individual stars and binaries, their mutual interaction with other cluster members and the dynamical evolution of the stellar system as a whole. With the GRAPE series of computers (Ebisuzaki et al. 1994) it is possible to tackle problems in which stellar evolution and stellar dynamics are interactively connected. We summarize a few specific problems that can be addressed with these models:

- Birth rate of $O B$ runaway stars.

An OB runaway is a main-sequence star which has an anomalously high space velocity compared to other $O$ and B stars in the galactic disc (Gies \& Bolton 1986). Two completely different scenarios have been proposed for their formation in young clusters: the $O B$ star is ejected from a close binary at the moment its companion explodes as a supernova (Blaauw 1992); or the OB runaway is ejected in a dynamical 3-body encounter (Leonard 1995).

- The origin of X-ray binaries.

A neutron star or a black hole accretes from a Roche lobe filling, less massive companion. The frequency of LMXBs in globular clusters can be explained by tidal capture (Fabian et al. 1975), but there are many doubts about the efficiency of this process (Ray 1987). Similar mechanisms 
apply to low-luminosity X-ray sources and cataclysmic variables, which are preferentially found in the cores of massive globular clusters with a high central density (Johnston \& Verbunt 1996). The discovery of close binaries in globular clusters has led to an alternative formation mechanism, exchange of a compact object into a pre-existing binary (Bailyn 1996).

- Dynamical formation of triple systems.

The millisecond pulsar PSR B1620-26 in the core of the globular cluster M4 is possibly a triple system (Rasio et al. 1995). An optical counterpart has been proposed by Bailyn et al. (1994); it is a low-mass main-sequence star, which accompanies the neutron-star plus white-dwarf binary. Dynamical formation mechanisms are inevitable.

- Colour gradients in globular-cluster cores.

Post-collapse globular clusters tend to be bluer in the centre than in the outskirts. This colour gradient cannot be readily explained by mass segregation alone. An additional source of red stars in the cluster halo together with an extra source of blue light in the cluster centre are required to explain the observed colour gradients (Djorgovski et al. 1991). Related problems are the significant depletion of subgiants in the cores of dense clusters and the concentration of blue stragglers there when compared to horizontal branch stars (Paresce et al. 1991).

- Ejection of compact objects

The concept of an intrinsic asymmetry in supernovae, ejecting the newly formed compact object with a ligh velocity, is vividly debated by upholders and antagonists from various fields of astronomy. The modest escape velocities of globular clusters make them excellent laboratories for studying the kick velocity distribution. The effect of the presence or absence of compact objects affects the dynamical evolution (Kim et al. 1997) and the binary population (Hut 1994) of a star cluster considerably.

- Formation of black holes in galactic nuclei.

The apparent central velocity dispersion in the cores of several dense star clusters and the results from spectroscopic observations of nearby galaxies suggests the presence of a massive central black hole (Kormendy et al. 1996, Ford et al. 1994 and Harms et al. 1994). The: formation of a central black hole may result from runaway growth of a black hole following multiple collisions with other cluster members (Quinlan et al. 1995, Lee 1995).

\section{Acknowledgements}

This work was supported in part by the Netherlands Organization for Scientific Research under grant PGS 13-4109, by Spinoza grant 08-0 to E. P. J. van den Heuvel, and by the Leids Kerkhoven Boscha Fonds. SPZ thanks the Institute for Advanced Study and the University of Tokyo for their hospitality. CAT is very grateful to Donglas Heggie and the UK PPARC for supporting his attendance from their HARP grant.

\section{References}

Bailyn, C. D. 1996, in E. Milone, J.-C. Mermilliod (eds.), The origins, evolution, and destinies of binaries in clusters, Vol. 90, A.S.P. Conf. Ser, p. 320

Bailyn, et al. C. D., Rubinstein, E. P., Girard, T. M., Dinescu, D. I., Rasio, F. A., Yanny, B. 1994, Ap.J, 433, L89

Blaauw, A. 1992, in Massive Stars and the Interstellar Medium, Wisconsin

Chernoff, D., Weinberg, M. 1990, ApJ, 351, 121

de la Fuente Marcos, R. 1996, A\&A 314, 453

Djorgovski, S., ct al. Piotto, G., Chernoff, D., Phinney,E. S. 1991, Ap.J, 372, L41

Ebisuzaki, T., ct al. Makino, J., Fukushige, T., Taiji, M., Sugimoto, d., Ito, t., Okumura, S. K. 1993, Publ. A.str. Soc. Japan, 45,269

Einsel, Ch., Spurzem, R., 1997, astro-ph/9704284

Fabian, A., Pringle, J., Rees, M. 1975, MNRAS, 172, 15p

Ford, H. C, et al. Harms, R. J., Tsvetanov, Z. I., Hartig, G. F., Dressel, L. L., Kriss, G. A., Bohlin, R. C., Davidsen A. F., Margon, B., Kochhar, A. K. 1994, Ap.J, 435, L27

Fukushige, T., Heggie, D. C. 1995, MNRAS, 276, 206

Gies, D. C., Bolton, C. T. 1986, Ap.JS, 61, 491

Harms, R. J., Ford, H. C., Tsvetanov, Z. I., Hartig, G. F., Dressel, L. L., Kriss, G. A., Bohlin, R., Davidson, A. F. Margon, B.. Kochhar, A. K. 1994, Ap.J, 435, L35

Heggic, D. C., 1992, Nat, 359, 772

Hut, P. 1994, IAU 165, in Compact stars in binaries, eds. J. van Paradijs and E. P. J. van den Henvel \& E. Kunlkers, Kluwer, p. 377

Hut, P., Makino, J. \& McMillan, S. 1995, Ap,J 443, 93 
Hut, P., et al. McMillan, S., Goodman, J., Mateo, M., Phinney, S., Pryor, C., Richer, H., Verbunt, F., Weinberg, M. 1992, PASP, 104, 981

Johnston, H. M., Verbunt, F. 1996, A\&A, 312, 80

Kim, S. S., et al. 1997, ApJ, in press

Kim, S. S., Lee, H. M., Goodman, J. 1998, ApJ, in press.

Kormendy, J., Bender, R., Richstone, D., Ajhar, E. A., Dressler, A., Faber, S. M., Gebhardt, K., Grillmair, C., Lauer, T. R., Tremaine, S. 1996, ApJ, 459, L57

Kulkarni, S. R., Hut, P., McMillan, S. 1993, Nature, 364, 421

Lee, H. M. 1987, Ap. J, 319, 772

Lee, H. M. 1995, MNRAS 272, 605

Leoliard, P. J. T. 1994, MNRAS, 277, 1080

Meylan, G., Heggie, D. C. 1997, astro-ph/9610076

Paresce, F., et al. Shara, M., Meylan, G., Baxter, D., Greenfield, P., Jedrzejewski, R., Nota, A., Sparks, W., Albrecht, R., Barbieri, C., Blades, J., Boksenberg, A., Crane, P., Deharveng, J., Disney, M., Jakobsen, P., Kamperman, T., King, I, Macchetto, F., Mackay, C., Weigelt, G. 1991, Nat, 352, 297

Portegies Zwart, S. F., Verbunt, F. 1996, A\&A, 309, 179

Portegies Zwart, S. F., Hut, P., McMillan, S., Verbunt, F. 1997, astro-ph/9706090

Quinlan, G. D., Hernquist, L., Sigurdsson, S. 1995, ApJ, 440, 554

Rasio, F. A., McMillan, S., Hut, P. 1995, ApJ, 438, L33

Ray, A., Kembhavi, A., Antia, H. 1987, A\&A, 184, 164

Spurzem, R., Giersz, M. 1996, MNRAS, 283, 805

Spurzem, R., Aarseth, S. J. A. 1996, astro-ph/9605003

Tout, C. A., Aarseth, S. J. A., Eggleton, P. P., Pols, O. R., 1997. MNRAS, in press 